\title{
Tests of Timing Properties of Silicon Photomultipliers
}

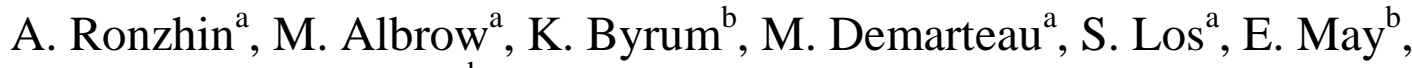 \\ E. Ramberg ${ }^{\mathrm{a}}$, J. Va'vra ${ }^{\mathrm{d}}$, A. Zatserklyaniy ${ }^{\mathrm{c}}$ \\ ${ }^{a}$ Fermi National Accelerator Laboratory, Batavia, IL 60510, USA \\ ${ }^{\mathrm{b}}$ Argonne National Laboratory, Argonne, IL 60637 USA \\ ${ }^{\mathrm{c}}$ University of Puerto Rico, Mayaguez, Puerto Rico 00681 USA \\ ${ }^{\mathrm{d}}$ SLAC National Accelerator Laboratory, Stanford, USA
}

\begin{abstract}
Timing measurements of Silicon Photomultipliers (SiPM) at the picosecond level were performed at Fermilab. The core timing resolution of the electronic measurement technique is approximately 2 picoseconds. The single photoelectron time resolution (SPTR) was measured for the signals coming from the SiPM's. A SPTR of about one hundred picoseconds was obtained for SiPM's illuminated by laser pulses. The dependence of the SPTR on applied bias voltage and on the wavelength of the light was measured. A simple model is proposed to explain the difference in the SPTR for blue and red light. A time of flight system based on the SiPM's, with quartz Cherenkov radiators, was tested in a proton beam at Fermilab. The time resolution obtained is 35 picoseconds per SiPM. Finally, requirements for the SiPM's temperature and bias voltage stability to maintain the time resolution are discussed.
\end{abstract}

\section{Introduction}

Time-of-flight (TOF) measurement is a very useful technique to distinguish between subatomic particles. To make an identification of a particle species means to define the particle mass. This is possible by measuring the particle momentum from the radius of curvature in a magnetic field and the particle velocity measured by TOF. Typical TOF resolutions have been on the order of 100 picoseconds (ps). Partly, this is due to the use of scintillators for light generation, which have extended emission times. Partly this is due to the inherent limitations of large size photomultiplier tubes, whose intrinsic SPTR is large.

The SiPM's produced by Hamamatsu (termed Multi Pixel Photon Counters, or MPPC) could be an option for upgrading capabilities of TOF systems. These MPPC's are blue sensitive photosensors, matching well to the light from scintillators and Cherenkov radiators. As we discuss below, the transit time spread of the MPPC is about $100 \mathrm{ps}$ for a single photoelectron. The blue photon detection efficiency of the devices can be about $65 \%$. MPPC's with a sensitive area of $3 \times 3 \mathrm{~mm}^{2}$ are on the market. We discuss here measurements of several SiPM's timing characteristics performed at the Fermi National Accelerator Laboratory (Fermilab). The first test beam results are also presented. 


\section{Setups to Study SiPM Timing}

Two setups at the Silicon Detector Facility at Fermilab were created for studying SiPM timing characteristics (see Figure 1). The first one contains a dark box containing the SiPM's under study. The elements of the setup are a picosecond-level pulse generator (Picosecond Pulse Lab, model 2000) used as a driver for a fast blue light emitting diode (LED), and a SiPM connected to an Ortec VT120 fast preamplifier and Ortec 934 Constant Fraction Discriminator (CFD). The LED light level was changed by a rotating wheel with light attenuation filters and monitored by PIN diodes. The LED attenuated light was delivered to the SiPM's by an optical fiber. The SiPM's housing was mounted on a Peltier element which was used to change and stabilize the SiPM's temperature. The Peltier allowed controlling the temperature in the range of $0 \mathrm{C}$ to $+25 \mathrm{C}$ with $0.5 \mathrm{C}$ of accuracy. The trigger out of the pulse generator was used as the timing start signal and the CFD signal as the stop. The time difference between the start and the stop signals was measured by an Ortec 567 Time to Amplitude Converter (TAC), whose analog output was fed into an Ortec AD114 14-bit Analog to Digital Converter (ADC), located in a CAMAC crate with PC readout. This system had a dynamic range of $50 \mathrm{~ns}$, with a single channel resolution of 3.1 ps.

The second setup also consisted of a dark box where SiPM's were placed. The SiPM output was connected to an Ortec 9327 module which had a preamplifier and CFD combined in a single unit. An Ortec VT120 preamplifier or attenuator was used between the SiPM output and 9327 input, when needed. The SiPM's were illuminated by a PiLas laser (635 nm and $405 \mathrm{~nm}$ heads with light pulse duration of $34 \mathrm{ps,} \mathrm{FWHM).} \mathrm{The} \mathrm{PiLas}$ allowed change of the light intensity in the dynamic range $0-100 \%$. We monitored the laser light by a PIN diode and a small photomultiplier. A Peltier element was also used to stabilize the SiPM's temperature, but was later replaced by a Ranque-Hilsch vortex tube to increase the temperature dynamic range and improve the temperature stability. The range of temperature is $+25 \mathrm{C}$ to $-20 \mathrm{C}$ inside the dark box. Four thermocouples were installed in different places inside and outside the box to achieve better temperature control. The temperature could be changed, stabilized and monitored with about $0.1 \mathrm{C}$ accuracy by hardware and software managed by a PC using Lab View software. The PiLas trigger signal is used as the timing start and the 9327 NIM out signal is used as the timing stop. The timing measurement is the same as with the LED setup. A Keithley supply was used for the SiPM's as a bias supply. The unit allows the supply voltages to be maintained with $10 \mathrm{mV}$ accuracy.

In all measurements discussed below, we report timing resolutions as the sigma of a Gaussian fit to the timing distribution and refer to them as SPTR. The SPTR refers to single photoelectron measurements.

\section{Establishing Conditions for Testing SiPM Time Resolution}

Schematics of the initial test are shown in Figure 2. To begin with, the start and stop signals were delivered to the TAC567 and AD114 from the same generator (Figure 2a). The contribution to the time resolution from the electronics, measured in this way, was 2 ps. The calibration of the absolute measurement (3.1 ps per ADC channel) was obtained by introducing a 1000 ps delay into the stop signal. The "warming up" time of 
the TAC567 plus AD114 is about 20 minutes. After half an hour the peak position is stable within $+/-1.5$ ps during the time of each measurement at room temperature (25 C).

The next test was performed with a Hamamatsu MPPC with $1 \mathrm{x} 1 \mathrm{~mm}^{2}$ sensitive area, illuminated by an intense LED. The driver pulse was used as a start signal and the MPPC as a stop signal (Figure 2b). The amount of LED light was enough to observe a time resolution of about 10 ps. The mean timing peak position with respect to temperature and bias voltage was measured. A shift of 11.5 ps per 0.5 degree $C$ (at ambient room temperature of 24 degree C) was measured. An overvoltage of 1 Volt was applied to the MPPC (where overvoltage refers to voltage above the depletion voltage of the photodiode). A 6.2 ps time shift per $10 \mathrm{mV}$ of change around the overvoltage was detected. These preliminary tests provided the conditions needed to keep a stability of less than 10 ps in time resolution. We attempted to maintain these conditions in future tests.

A few tests were performed to evaluate the working Ortec 9327 dynamic range. The unit timing parameters depend on pulse duration and amplitude, according to the Ortec specifications. The 9327 unit should accept pulse widths up to 5 nsec full width at half maximum. A simple clipping circuit was used to shorten the MPPC pulses (Figure 3). The 9327 has an internal jumper which allows operation in one of two ranges of accepted signal amplitude: $0-30 \mathrm{mV}$ or $1-150 \mathrm{mV}$. A first test was performed to see how the 9327 time resolution depended on bias voltage. This measurement was performed with a Hamamatsu 3x3mm ${ }^{2}$ MPPC, with an Ortec VT120 leading into the Ortec 9327 ( $0-150 \mathrm{mV}$ range). Light pulses came from the $635 \mathrm{~nm}$ PiLas head, with about 100 photoelectrons. The result is shown in Figure 4.

Analogous studies were continued with another MPPC. An attenuator of $20 \mathrm{db}$ (FP-50 Texscan) was introduced into the signal line starting with 1.2 Volt of overvoltage (Figure 5) and the time resolution was measured with dependence of the signal amplitude. Note that reducing the amplitude in this way retains the best timing resolution for this device.

Subsequently, both amplitude ranges of the 9327 were tested. Attenuators were used to keep the MPPC amplitude in the central range for the CFD. The result is presented in Figure 6. Note that the timing resolution is not significantly dependent on which physical range of the 9327 is used. For further data taking, we tried to keep amplitudes from the MPPC's inside the $10-40 \mathrm{mV}$ range.

\section{The SiPM Timing Study}

We then compared some characteristics of the following SiPM devices:

- Hamamatsu 1x1 mm² area with $100 \times 100 \mu \mathrm{m}^{2}$ pixel size (100 total pixels)

- Hamamatsu 1x1 mm² area with 50x50 $\mathrm{mm}^{2}$ pixel size (400 pixels)

- Hamamatsu $1 \times 1 \mathrm{~mm}^{2}$ area with $25 \times 25 \mu^{2}$ pixel size (1600 pixels)

- Hamamatsu $3 \times 3 \mathrm{~mm}^{2}$ area with $50 \times 50 \mu^{2}$ pixel size (3600 pixels)

- IRST (Italy) $1 \times 1 \mathrm{~mm}^{2}$ area with $40 \mathrm{x} 40 \mu^{2}$ pixel size (625 pixels)

The SPTR dependence on the overvoltage were measured. Both $405 \mathrm{~nm}$ and $635 \mathrm{~nm}$ PiLas laser heads were used to perform the SPTR measurements. To ensure single photon registration on the surface, we demanded that more than $90 \%$ of the pulses resulted in 
pedestal (zero amplitude) events. We found that events with two or more detected photons gave negligible contributions to the SPTR. The stated time jitter due to the PiLas is 34 ps FWHM, which is negligible compared to the typical 100 ps SPTR.

The results for the MPPC $1 \times 1 \mathrm{~mm}^{2}$ devices are shown in Figure 7. The general trend is an improvement of the time resolution with an increasing overvoltage. Another point is that the SPTR is systematically better for the Hamamatsu devices with $635 \mathrm{~nm}$ PiLas illumination compared to $405 \mathrm{~nm}$. This effect is opposite to data obtained for the IRST device, which is shown in Figure 8.

Some tendency of improvement of the SPTR was observed for the smaller pixel size for the $1 \mathrm{x} 1 \mathrm{~mm}^{2}$ devices. A worse SPTR was measured for the Hamamatsu $3 \mathrm{x} 3 \mathrm{~mm}^{2}$ MPPC, with the dependence on light wavelength the same as for the smaller Hamamatsu devices (Figure 9).

An inverse square root dependence of the MPPC's time resolution was observed when the number of photoelectrons detected was increased (Figure 10). The number of photoelectrons was estimated on the basis of the single photoelectron's signal amplitude, which is perfectly defined for SiPM's[1].

Figure 11 presents dependence of time resolution, time delay change and signal amplitude on temperature for the $3 \times 3 \mathrm{~mm}^{2}$ MPPC illuminated by blue light (405 nm).

\section{Discussion}

The dependence of the absorption length versus the light wavelength in silicon is shown in Figure 12.[2] A picture of the electric field distribution for the shallow junction SiPM produced by IRST, Italy is presented in Figure 13.[3] For the IRST SiPM's, the n+ side of the silicon faces the light. One can see that if a photon absorbs close to the SiPM surface then the originated carriers will be holes. Likewise, the carriers will be electrons if the photon is absorbed deep into the silicon. The absorption length is about $100 \mathrm{~nm}$ for the $405 \mathrm{~nm}$ photon (blue light PiLas head) and $4 \mu \mathrm{m}$ for the $635 \mathrm{~nm}$ (red light PiLas head).

Thus, blue photons produce mostly holes, which travel to the high electric field and eventually develop an avalanche. The red photons produce mostly electrons traveling into the high field from the opposite direction. The mobility of holes in silicon's electric field is about 3 times less than for electrons, but the hole's path length is 40 times less. So the combined time spread of carriers originated by blue photons should be about one order of magnitude less than that originated by red photons. This could explain why the SPTR is better for blue light.

The magnitude of the velocity of carriers inside of silicon is about 1-10 ns per 300 $\mu \mathrm{m}$, depending on the electric field applied, silicon impurity and temperature.[4] According to a rough estimation, a spread on the order of a few microns of travel distance could provide $100 \mathrm{ps}$ of the corresponding time spread. This simple picture does not take into consideration the time jitter due to an avalanche development, lateral avalanche size, etc, but only considers the initial carrier's time spread.[5] Nevertheless, this naïve model describes reasonably the data obtained for the IRST SiPM.

For the Hamamatsu MPPC's, the $\mathrm{p}^{+}$side faces the light. The electrons are the carriers for the $405 \mathrm{~nm}$, and the holes are for the $635 \mathrm{~nm}$ in this case. If the SiPM is structured similarly to the IRST SiPM, then the time spread for the single photoelectron 
should be better when illuminated by $405 \mathrm{~nm}$ light. But the opposite was observed experimentally, i.e. the $1 \times 1 \mathrm{~mm}^{2}$ SiPM's show about $15 \%$ better SPTR for the $635 \mathrm{~nm}$ light. Almost no significant SPTR difference was measured for the $3 \times 3 \mathrm{~mm}^{2}$ devices for $405 \mathrm{~nm}$ and $635 \mathrm{~nm}$ illumination. It is probable that the location of the high field region would have an influence on the SPTR, according to the model presented above. One question that arises: where is the high field area for the Hamamatsu SiPM structure located? Another issue is that we do not know exactly the magnitude of the time jitter produced by electrons and holes inside of the silicon. We note that the measured difference in the SPTR for the SiPM's illuminated by $405 \mathrm{~nm}$ and $635 \mathrm{~nm}$ is at most $20 \%$.

Figure 8 shows some SPTR improvement with smaller SiPM pixel size. This effect is likely due to the SiPM's capacitance decrease. The worsening of the SPTR for $3 \times 3 \mathrm{~mm}^{2}$ device is also in agreement with the increased capacitance.

\section{Test beam data}

A high energy test beam facility exists in the Meson Detector Building at Fermilab. The beamline can be tuned for $120 \mathrm{GeV} / \mathrm{c}$ protons from Fermilab's Main Injector, or the beam can be brought onto a target providing lower momentum secondary beams.

When we first installed in the test beam in June, 2008, we had only two 3x3 mm² Hamamatsu MPPC's. Each of these devices were coupled with a $6 \times 6 \times 12 \mathrm{~mm}^{3}$ Cherenkov radiator, made of quartz. Two Keithley power supplies with a standard circuit board schematic applied the bias voltage. The ORTEC VT120 fast preamplifiers were used in series with the MPPC's output signals. Just as in the bench tests, the MPPC's amplified signals were delivered to the 9327 Ortec unit, followed by the time measurement of the TAC567/AD114 combination. A single ADC count in the AD114 corresponded to 3.1 ps. The full dynamic range was 50 ns.

The MPPC's with the Cherenkov radiators were aligned along the beam at normal incidence. A trigger counter was based on two small size PMT looking at a single scintillator with $2 \times 2 \mathrm{~mm}^{2}$ transverse size, $16 \mathrm{~mm}$ along the beam. The counter was located on a movable stage allowing alignment of the scintillator with $30 \mu \mathrm{m}$ accuracy. Both MPPC's and trigger counter were placed in a light-tight dark box with a copper coated G10 inside acting as an RF shield (Figure 14). The data were taken with a 120 $\mathrm{GeV} / \mathrm{c}$ proton beam. The overvoltage for each MPPC was 1 Volt. The gain of the MPPC's with this overvoltage is close to $0.3 \times 10^{6}$. We estimated the number of photoelectrons as about 15 for the MPPC's using the procedure described in [1]. The measured time resolution was about 70 ps per MPPC.

An attempt to improve the TOF resolution on a beam was performed during August, 2008. The same MPPC's with $3 \times 3 \mathrm{~mm}^{2}$ of sensitive area with optically attached Cherenkov radiators made of plexiglass $\left(3 \times 3 \mathrm{~mm}^{2}\right.$ transverse size) were tested with the $120 \mathrm{GeV} / \mathrm{c}$ proton beam. This time, with improved systematics, a time resolution of $48 \mathrm{ps}$ per SiPM was obtained, as shown in Figure 15. The number of photoelectrons was calculated to be 15 (Figure 16). We also tried the MPPC's with fiber optic face plates as Cherenkov radiators but the quality of the plate was poor and gave significantly poorer time resolution. 
A further run, with the goal of yet better timing, was performed during May-June 2009. This time we tested 8 SiPM's with quartz Cherenkov radiators of different lengths and shapes with mirrored side surfaces, cylindrical or rectangular in shape. The overvoltage used was up to 2 Volts. The best time resolution obtained was 35 ps per sample (Figure 17).

The conditions of this best performance were that we used a MPPC with $3 \times 3 \mathrm{~mm}^{2}$ of sensitive area, 50x50 $\mu$ m pixel size (3600 pixels). The Cherenkov radiator was made of fused silica, $3 \times 3 \mathrm{~mm}^{2}$ in transverse size, with either $6 \mathrm{~mm}$ or $8 \mathrm{~mm}$ in length (the same time resolution was observed for both), with mirrored side surfaces of the radiator. We used an optical grease (UV Dow Corning) between the SiPM and the radiators.

We believe that the time resolution we obtained with the Cherenkov radiators could be improved with a better design. It is possible to make two TOF counters based on SiPM's arranged as a plain matrix with Cherenkov radiators for the normally incident particles. The transverse size of the counters would then cover the beam. The thickness of each TOF counter would be of an order of a few mm including both the Cherenkov radiator and a silicon photomultipliers. Such a device would be relatively inexpensive, low in material budget and give much better performance in TOF than conventional scintillator counters.

\section{Summary of Results}

A new setup for timing measurements at the picosecond level has been studied at Fermilab. The core timing resolution of the amplifiers, discriminators and TAC/ADC combination is approximately 2 picoseconds. We made a study of single photoelectron time resolution for signals coming from Hamamatsu MPPC's and IRST SiPM's. The PiLas laser used blue $(405 \mathrm{~nm})$ and red $(635 \mathrm{~nm})$ heads for this study. We discussed the temperature and bias voltage stability level needed to maintain a few picoseconds time resolution. The SPTR improved with the overvoltage increase. The SiPM's time resolution is inversely proportional to the square root of the number of photoelectrons. A simple model is proposed to explain the difference in the SPTR when illuminating the silicon photomultipliers by $405 \mathrm{~nm}$ and $635 \mathrm{~nm}$ laser light. The SiPM's with $3 \times 3 \mathrm{~mm}^{2}$ of sensitive area and Cherenkov radiators were tested in a $120 \mathrm{GeV}$ proton beam at Fermilab. A best resolution of 35 ps per SiPM was obtained.

\section{Acknowledgements}

Our thanks to Henry Frisch of University of Chicago, for his long term support of this work and to Hogan Nguyen of Fermilab for technical support. 


\section{References}

1. A. Ronzhin, M. Demarteau, S. Los, E. Ramberg. Study of Timing Properties of Silicon Photomultipliers. FERMILAB - CONF- 09 - 055 - E - PPD, Preprint Fermilab, 2008.

2. K. Rajkanan, R. Singh, J. Shewchun. Solid State Electronics, 22, 793, 1979.

3. Claudio Piemonte. Development of Silicon Photomultipliers at IRST. FNAL report, October 25th 2006.

4. T. Ikoma, K. Hara. Temperature Dependence of Hole Saturation Velocity in Silicon. Applied Physics, 3, 431, 1974.

5. Lacaita A, Cova S, Spinelli, and Zappa F. Photon-assisted avalanche spreading in reach-through photodiodes. Applied Physics Letters. 62 (6), 8 February 1993. 


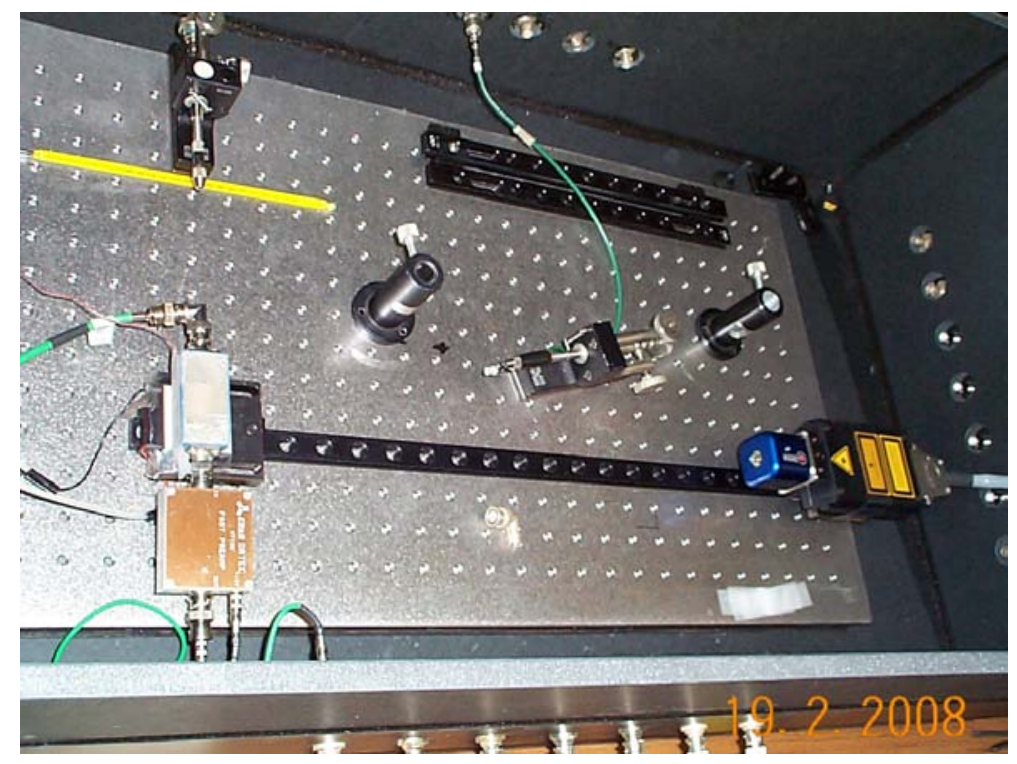

Figure 1. Setup for the SiPM timing study. The optical table is located in a dark copper shielded box. A picosecond Laser (PiLas) head is attached to the rail on the right side, and a SiPM box with Ortec preamplifier VT120 is on the left side. The LED is shown to one side.
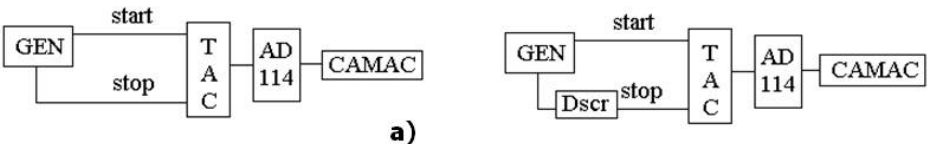

a)

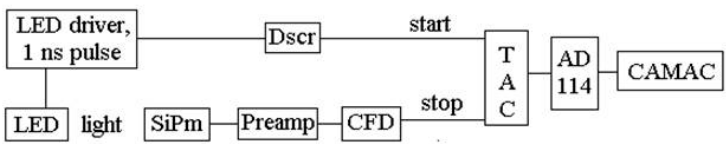

b)

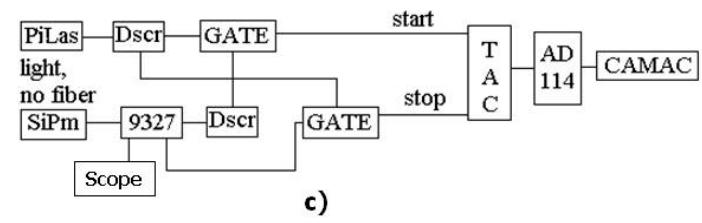

Figure 2. Schematics of the SiPM timing setup. Explanation is given in the text.

Abbreviations are as follows: TAC-time amplitude convertor, AD114-amplitude digital convertor, LED-light emitting diode, CFD 934-Ortec constant fraction discriminator, PiLas-picosecond laser, SiPM-silicon photomultiplier, 9327-1 GHz ORTEC amplifier and constant fraction timing discriminator. 


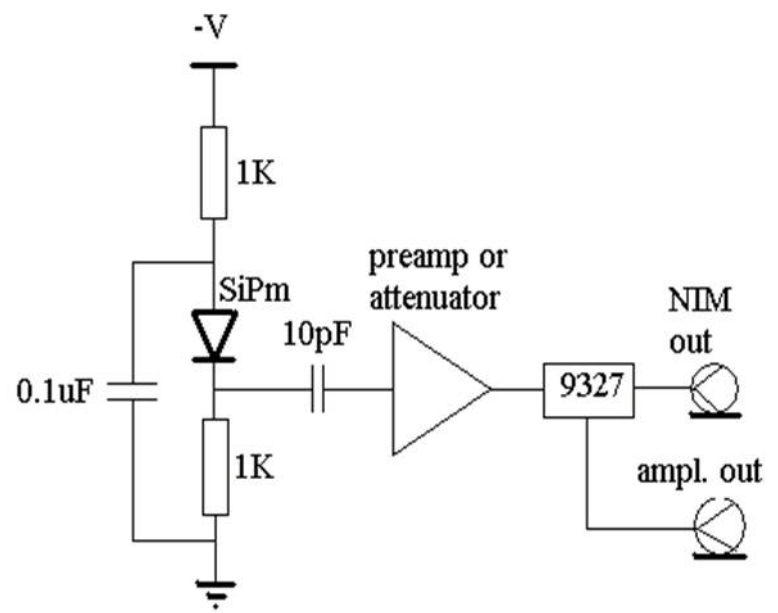

Figure 3. Schematic of the SiPM signal clipping circuit

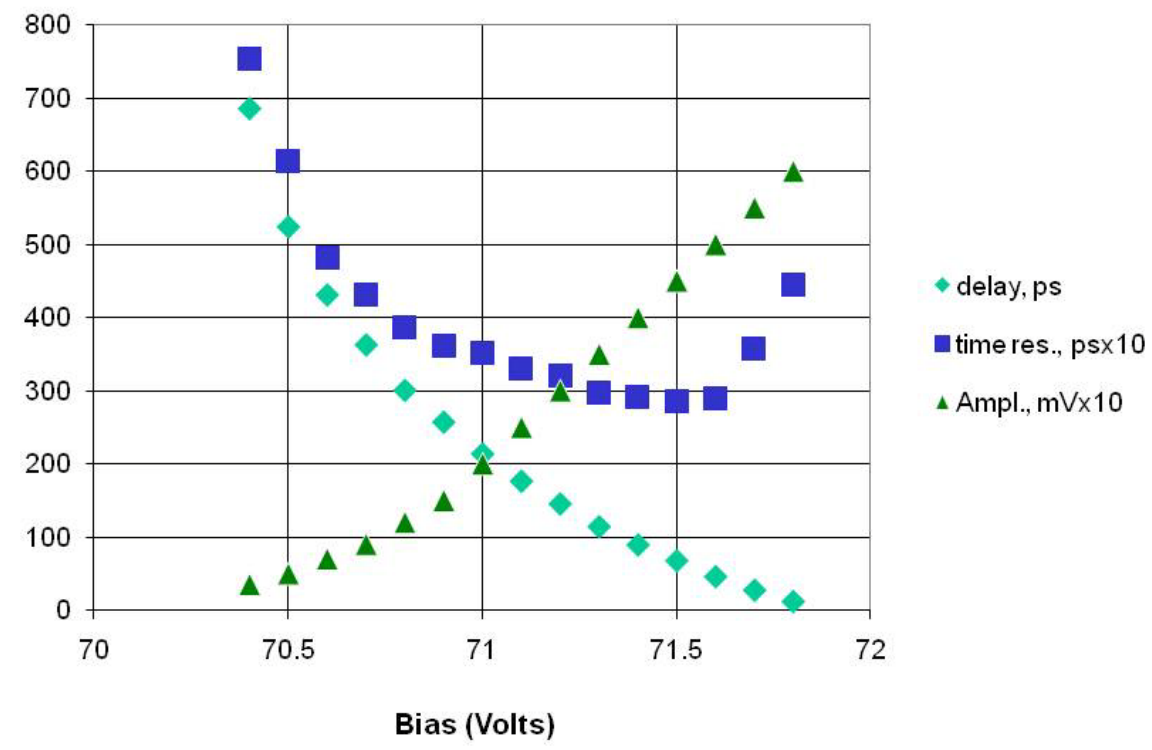

Figure 4. Response of the Hamamatsu $3 \times 3 \mathrm{~mm}^{2}$ MPPC to varying bias voltage. Response shown includes signal delay, signal amplitude and time resolution for approximately 100 photoelectrons. 


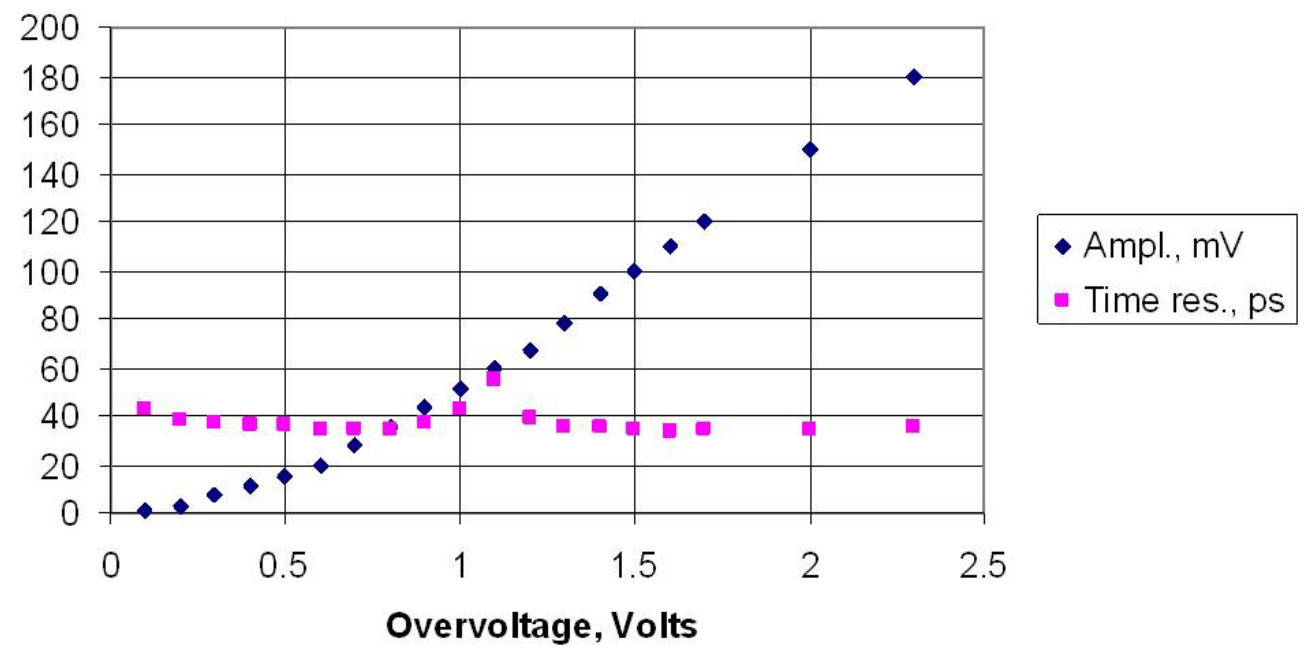

Figure 5. The MPPC signal amplitude and time resolution dependence on overvoltage for approximately 100 photoelectrons. A $20 \mathrm{db}$ attenuator was placed inline at $1.2 \mathrm{~V}$ overvoltage, showing the stability of the timing resolution using this method.

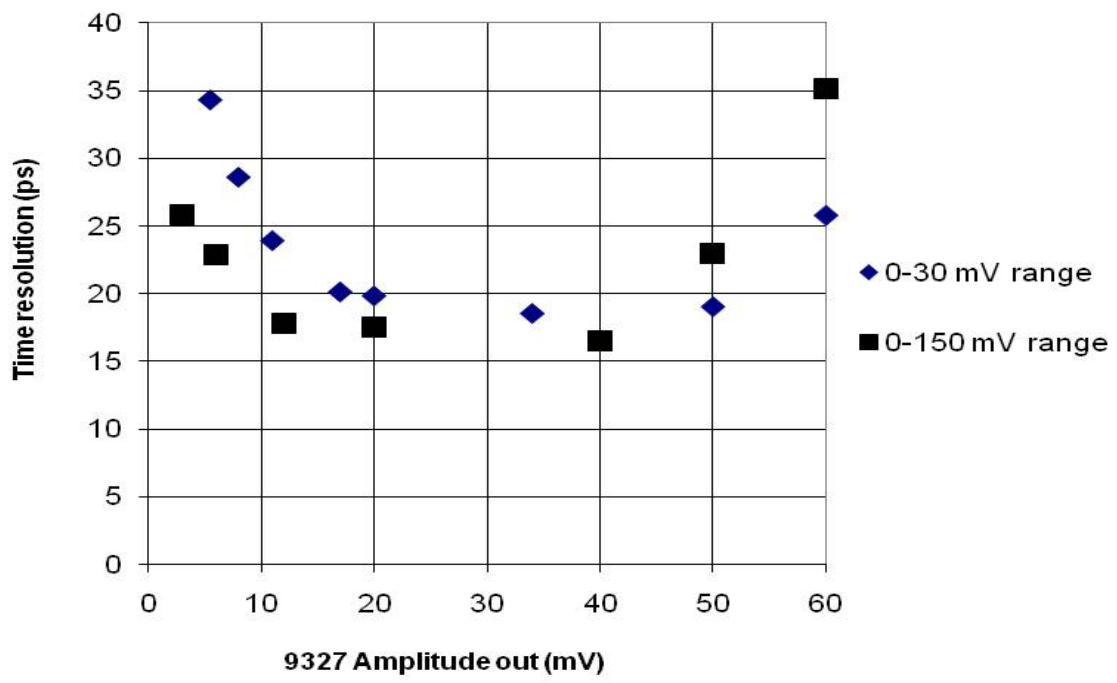

Figure 6. The MPPC time resolution (in ps for 100 photoelectrons), as a function of the signal amplitude for both range selections of the Ortec 9327 CFD. 


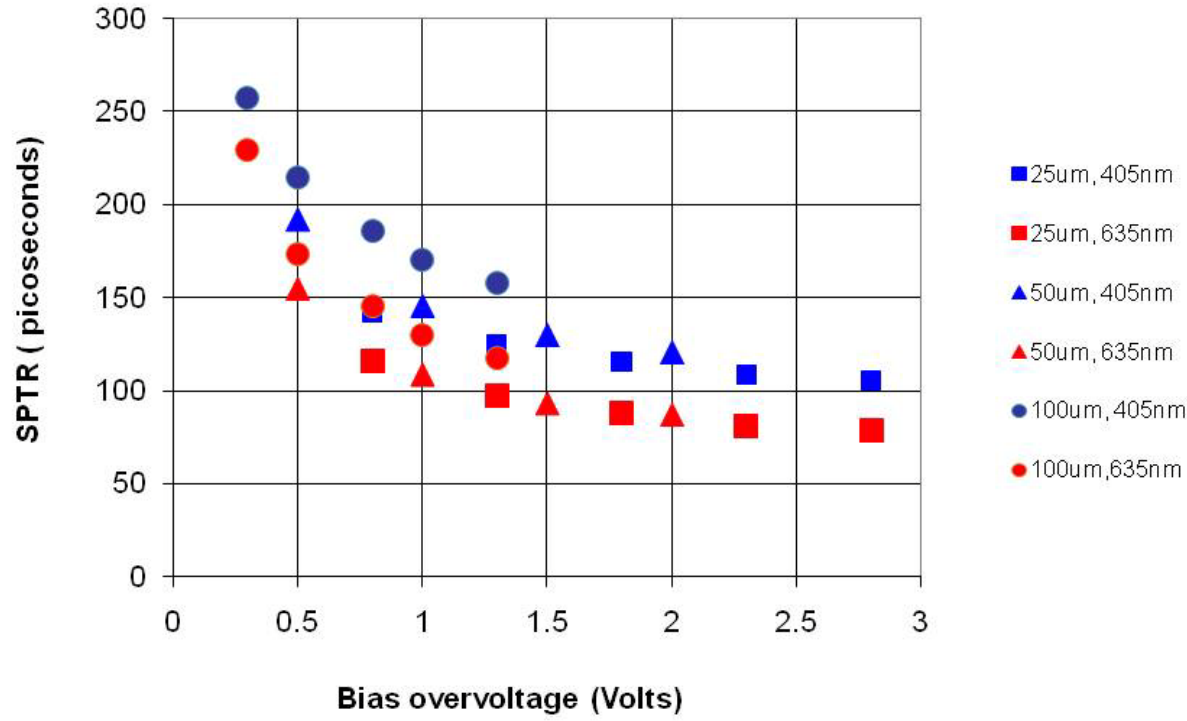

Figure 7. SPTR of various MPPC's (1x1 $\mathrm{mm}^{2}$ sensitive area) illuminated by blue (405 $\mathrm{nm})$ and red $(635 \mathrm{~nm})$ PiLas light vs. overvoltage.

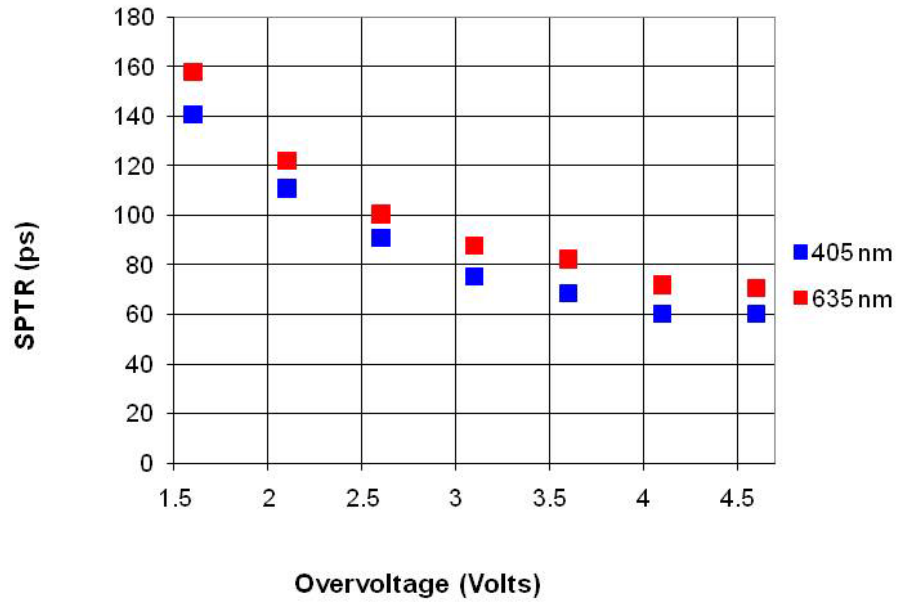

Figure 8. SPTR of the IRST SiPM (1x1 $\mathrm{mm}^{2}$ sensitive area) illuminated by blue and red laser light vs. overvoltage. 


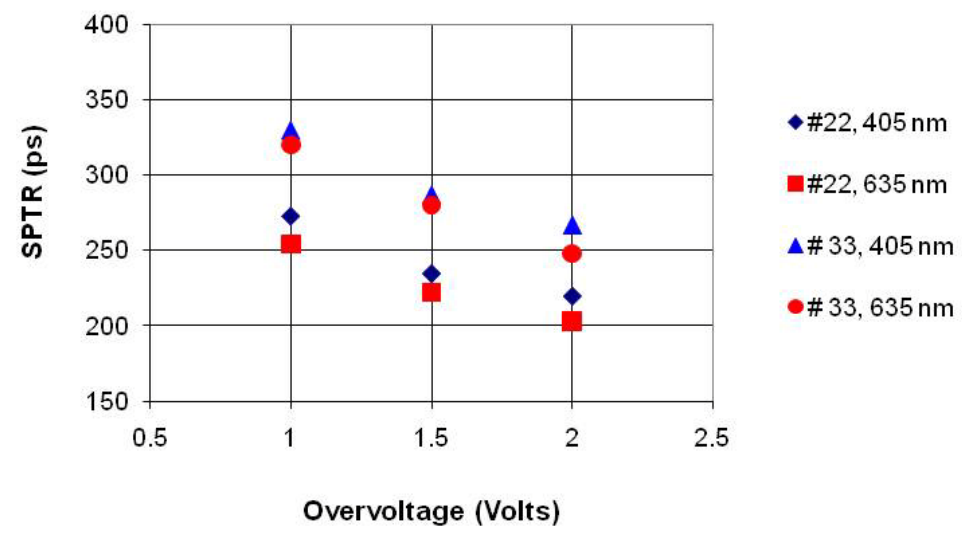

Figure 9. SPTR of two MPPC samples (3x3 $\mathrm{mm}^{2}$ sensitive area) illuminated by blue and red PiLas laser light.

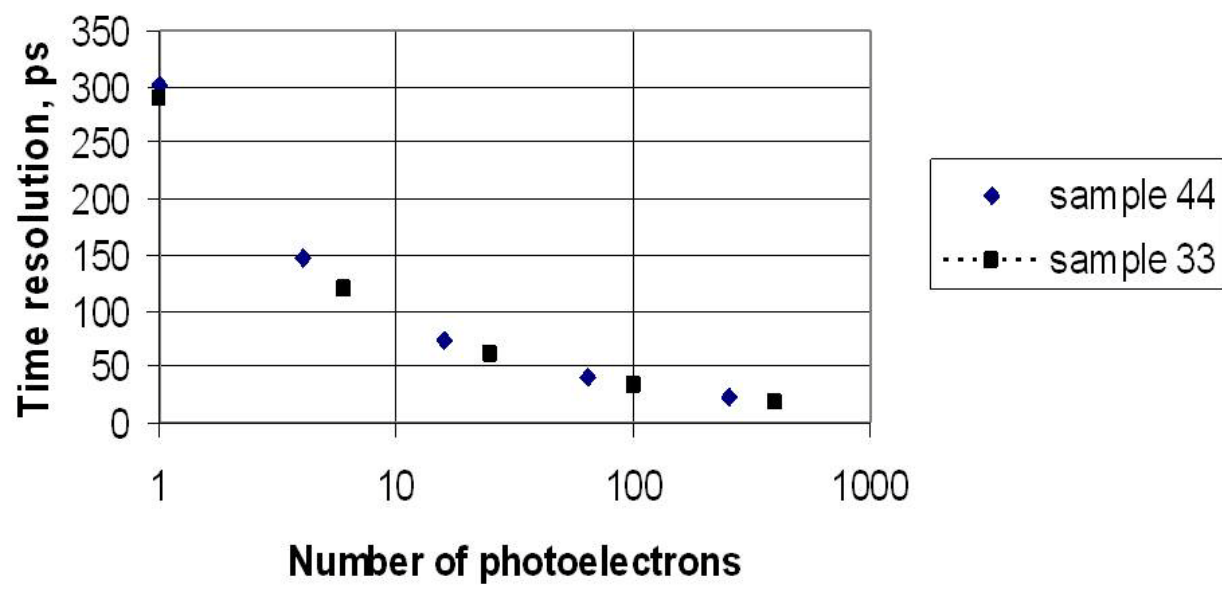

Fig. 10. Time resolution (sigma) of two MPPC samples versus the number of photoelectrons. 


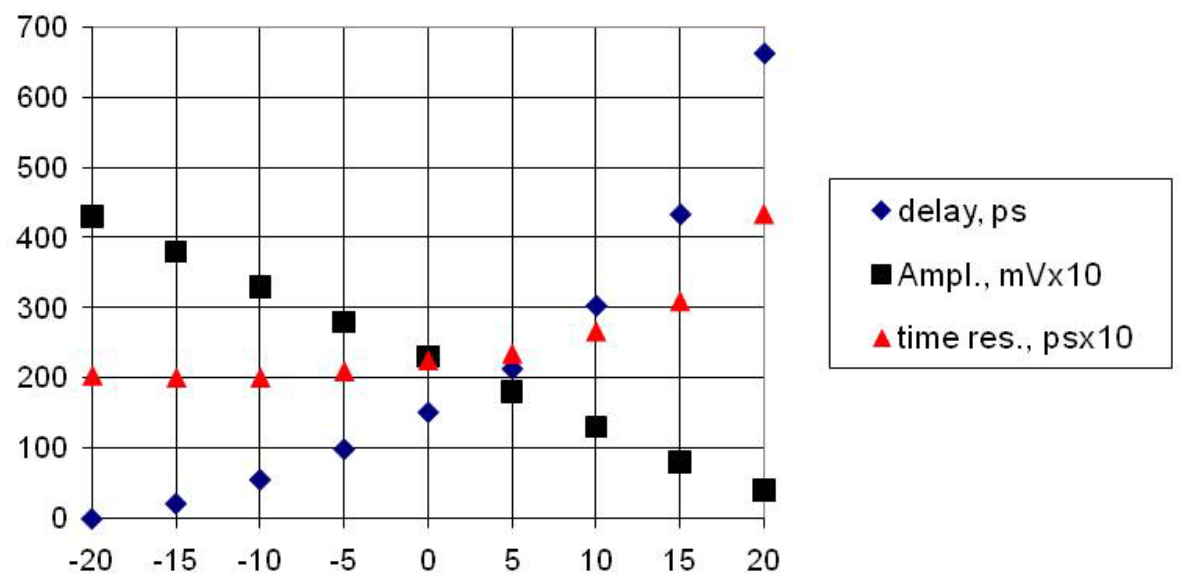

Temperature, degree C.

Figure 11. The MPPC signal delay, signal amplitude, and time resolution vs. temperature.

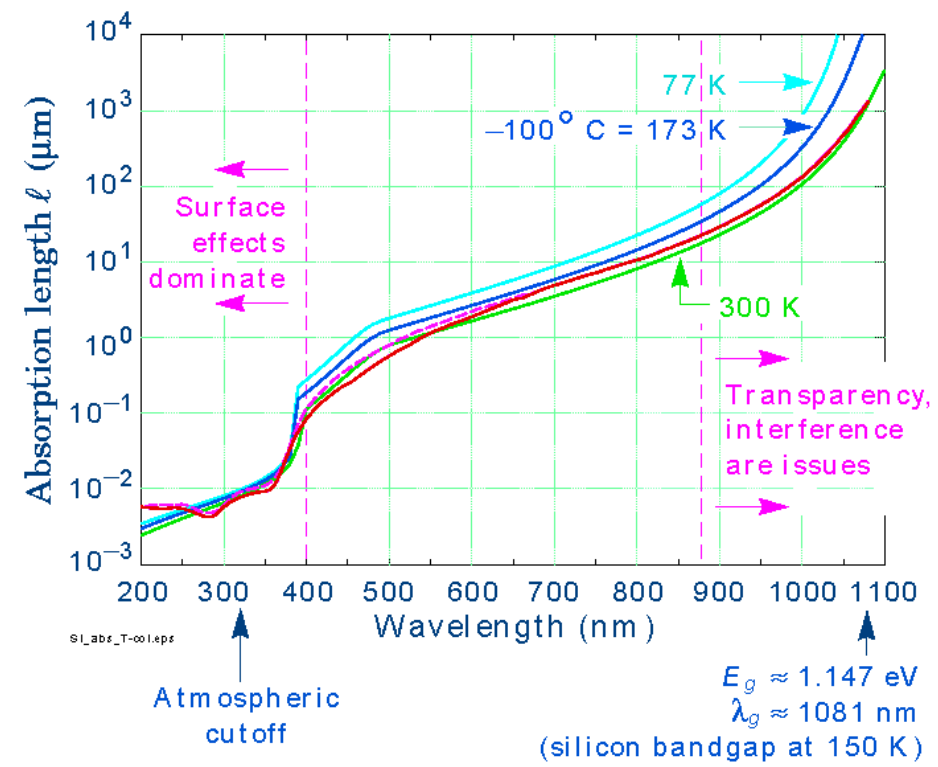

Figure 12. Absorption length in silicon as a function of photon wavelength. Different colored curves correspond to different temperature. [2] 


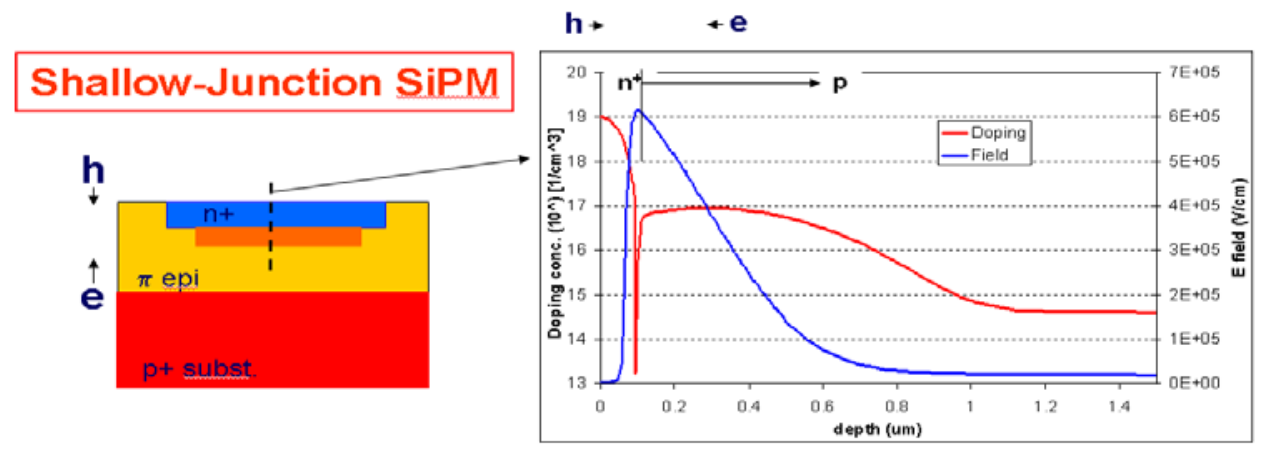

1) Substrate: p-type epitaxial

2) Very thin $n+$ layer

3) Quenching resistance made of doped polysilicon

4) Anti-reflective coating optimized for $\lambda \sim 420 \mathrm{~nm}$

Claudio Piemonte

FNAL

October $25^{\text {th }} 2006$

Figure 13. Distribution of the electric field and doping inside of IRST SiPM (top right) and the shallow-junction SiPM structure (left side of the drawing). Data presented by Claudio Piemonte at Fermilab. [3]

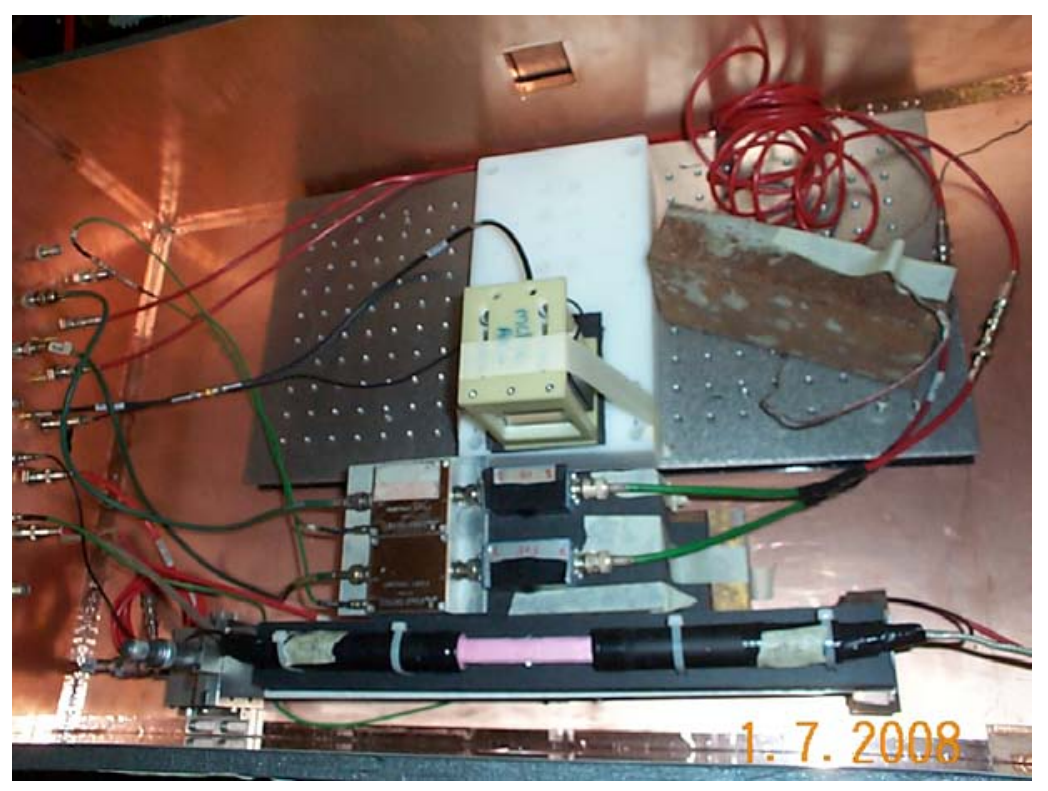

Figure 14. The setup at the beam test, with $120 \mathrm{GeV}$ protons entering from the bottom. A light tight lid is installed during beam delivery. The coppered G10 acts as RF shielding. Two SiPM boxes are shown, with the Ortec VT120 preamplifiers attached. A trigger counter with 2 PMTs is placed upstream, and a PMT with micro channel plates (MCP) is located downstream for timing verification. 


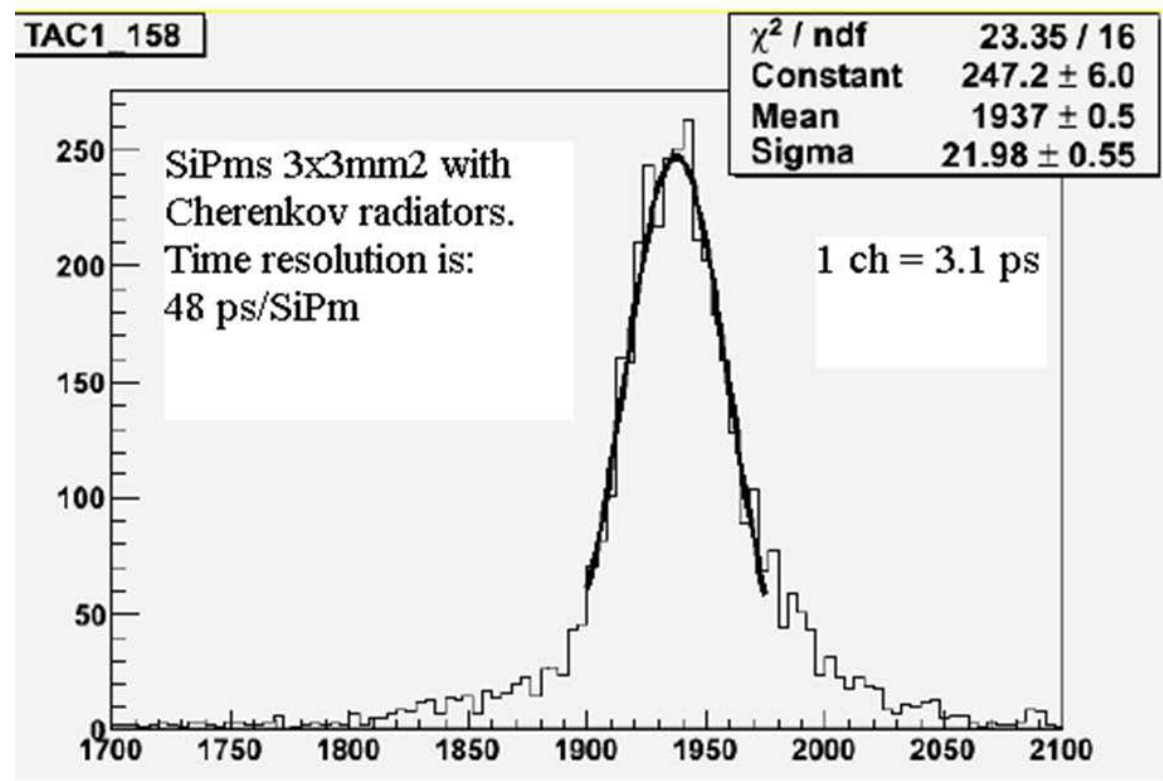

Figure 15. Timing difference spectrum for signals coming from two Hamamatsu $3 \times 3$ $\mathrm{mm}^{2}$ MPPC devices. Cherenkov radiators are attached. $120 \mathrm{GeV}$ protons are used for this test. Assuming identical responses from each device, the time resolution is 48 ps per SiPM.

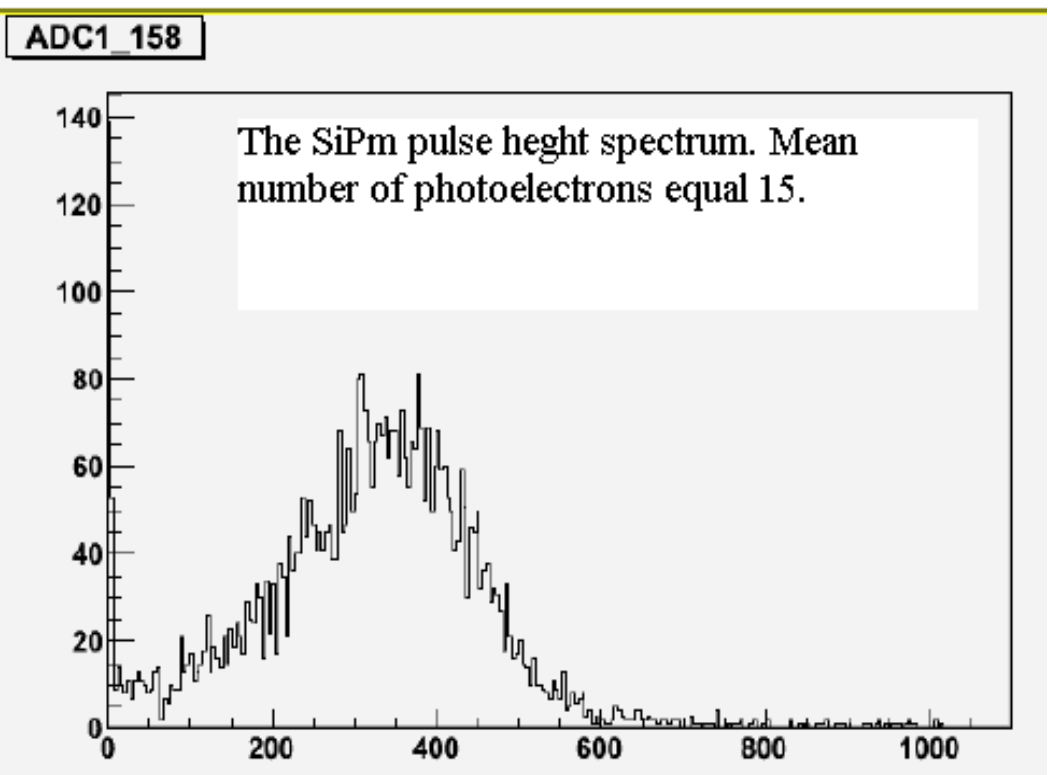

Fig. 16. The SiPM pulse height spectrum, obtained with120 GeV protons. 


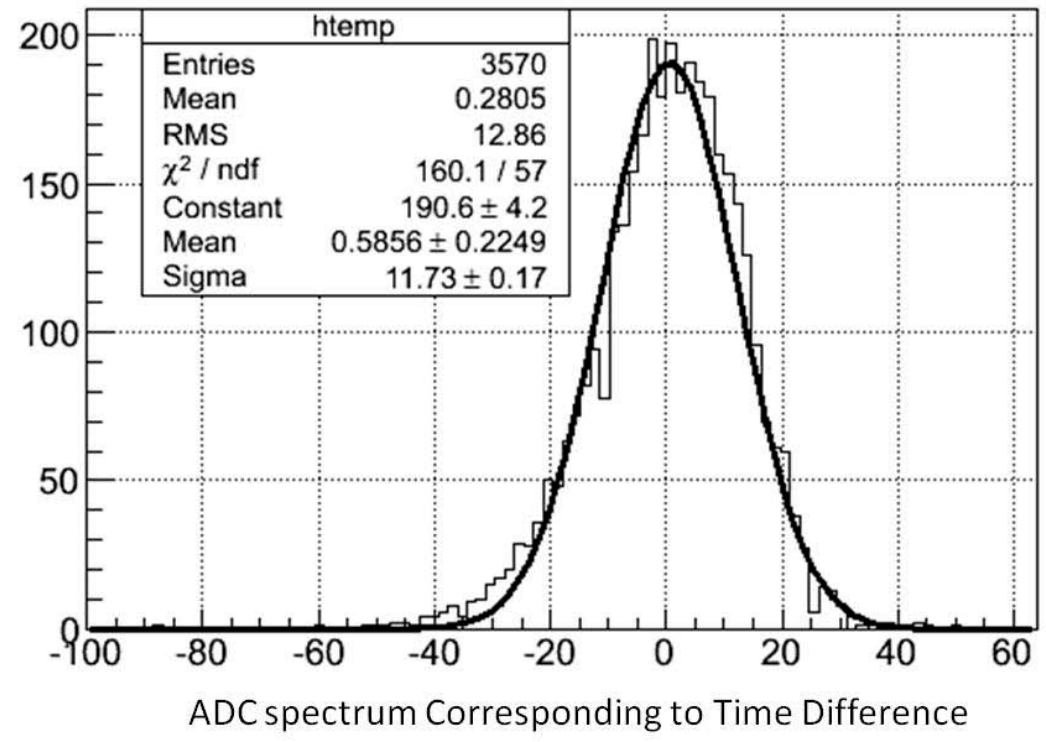

Fig. 17. Timing spectrum, obtained with $120 \mathrm{GeV}$ protons. Devices used were MPPC's (3x3 mm2) with Cherenkov radiator as start counter and a Photek 240 (normal particle incidence) as a stop counter. Time resolution (sigma) is 11.7 channels, or 35 ps, per SiPM. 